\title{
Study of Irradiations to Enhance the Induces the Dissociation of Hydrogen Bonds between Peptide Chains and Transition from Helix Structure to Random Coil Structure Using ATR - FTIR, Raman and ${ }^{1}$ HNMR Spectroscopies
}

\author{
A Heidari* \\ Faculty of Chemistry, California South University, 14731 Comet St. Irvine, CA 92604, USA
}

\section{Editorial}

Human tissues are connective tissues which are surrounded the surface of the kidney. They consist of Osteoid (Collagen containing component of bone) fibers (67\% Osteoid Type (I) and 33\% Osteoid Type (III)), Lysyl Oxidase and LOXL1, LOXL2, LOXL3, LOXL4 fibers in Collagen formation, Hydrolyzed Collagen (a common form in which Collagen is sold as a supplement) fibers, Collagenase (the enzyme involved in Collagen breakdown and re-modelling) fibers and Sponge (cell types) fibers among elastic fibers (Figures 1-6). These tissues are used as graft, since Collagenous tissues begin to be denatured after excision; chemical physics methods like Infrared (IR) light are used to improve chemical physics properties. In the current editorial, the spectroscopic analysis was performed associated with the Attenuated Total Reflectance Fourier Transform Infrared Spectroscopy (ATRFTIR), Raman and 1HNMR spectroscopies to evaluate the effect of Near-Infrared (NIR) or IR-A, Short-Wavelength Infrared (SWIR) or IR-B, Mid-Wavelength Infrared (MWIR) or IR-C, Intermediate Infrared (IIR) or IR-C, Long-Wavelength Infrared (LWIR) or IR-C and Far-Infrared (FIR) lights on the tissues, respectively [1-9].

Samples from human tissues were irradiated using thirty six Near-Infrared (NIR) or IR-A, Short-Wavelength Infrared (SWIR) or IR-B, Mid-Wavelength Infrared (MWIR) or IR-C, Intermediate Infrared (IIR) or IR-C, Long-Wavelength Infrared (LWIR) or IR-C and Far- Infrared (FIR) 100 (W) lamps for 24, 48, 72, 96, 120, 144 and $168 \mathrm{~h}$, respectively. In frequencies sweep test, dynamic force with linear and non-linear amplitudes, which are derived from strain sweep test, and frequencies range from $10 \mathrm{~Hz}$ to $110 \mathrm{~Hz}$ are induced and the corresponding parameters such as the storage and loss modulus are measured. The Attenuated Total Reflectance Fourier Transform Infrared Spectroscopy (ATR-FTIR), Raman and 1HNMR spectroscopies are used in order to observe the changes of molecular structures of these tissues. The effect of Near-Infrared (NIR) or IRA, Short-Wavelength Infrared (SWIR) or IR-B, Mid- Wavelength Infrared (MWIR) or IR-C, Intermediate Infrared (IIR) or IR-C, LongWavelength Infrared (LWIR) or IR-C and Far-Infrared (FIR) lights was seen by noticing the changes of the Amide bands in absorption spectra of tissues, respectively [10-19].

Storage and loss modulus reach their maximum values under 24 hours Near-Infrared (NIR) or IR-A, Short-Wavelength Infrared (SWIR) or IR-B, Mid-Wavelength Infrared (MWIR) or IR- C, Intermediate Infrared (IIR) or IR-C, Long-Wavelength Infrared (LWIR) or IR-C and Far- Infrared (FIR) irradiations to emphasize the cross link formation in the samples and the subsequent reduction after $48 \mathrm{~h}$ and $72 \mathrm{~h}$, respectively. Increasing the irradiations' doses, the processes of degradation predominate to cause the stiffness drop of the samples. In Attenuated Total Reflectance Fourier Transform Infrared Spectroscopy (ATR-FTIR), Raman and 1HNMR spectroscopies, changes in Amide (I) and (II) bands indicate changes in secondary structure of Osteoid fibers, Lysyl Oxidase and LOXL1, LOXL2, LOXL3, LOXL4 fibers, Hydrolyzed Collagen fibers, Collagenase fibers and
Sponge fibers (Figures 7-9). A notable shift of Amide (I) and (II) were observed to higher and lower frequencies, respectively (Figures 7-9).

Limited exposure to Near-Infrared (NIR) or IR-A, ShortWavelength Infrared (SWIR) or IR- B, Mid-Wavelength Infrared (MWIR) or IR-C, Intermediate Infrared (IIR) or IR-C, LongWavelength Infrared (LWIR) or IR-C and Far-Infrared (FIR) lights generate free radicals in aromatic amino acids, proteins or nucleic acids to form Osteoid molecules, Lysyl Oxidase and LOXL1, LOXL2, LOXL3, LOXL4 molecules, Hydrolyzed Collagen molecules, Collagenase molecules and Sponge molecules cross-linking [1]. It occurs in 24, 48 and $72 \mathrm{~h}$ of the irradiations to enhance the induces the dissociation of Hydrogen bonds between peptide chains and transition from helix structure to random coil structure has happened and so Amide (I) band has shifted to the higher frequencies. Furthermore, peptide bonds are broken and Osteoid denaturation, Lysyl Oxidase and LOXL1, LOXL2, LOXL3, LOXL4 denaturation, Hydrolyzed Collagen denaturation, Collagenase denaturation and Sponge denaturation make it shift in Amide (II) bands toward lower frequencies. This denaturation of Osteoid, Lysyl Oxidase and LOXL1, LOXL2, LOXL3, LOXL4, Hydrolyzed Collagen, Collagenase and Sponge cause loosing of chemical physics properties, accordingly.

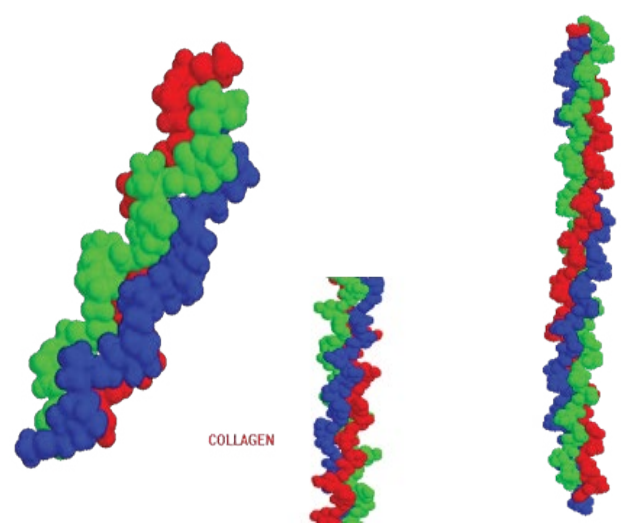

Figure 1: Different schematics of Collagen triple helix structure [1].

*Corresponding author: A Heidari, Faculty of Chemistry, California South University, 14731 Comet St. Irvine, CA 92604, USA, E-mail: Scholar.Researcher. Scientist@gmail.com

Received March 14, 2016; Accepted March 15, 2015; Published March 19, 2016

Citation: HeidariA (2016) Study of Irradiations to Enhance the Induces the Dissociation of Hydrogen Bonds between Peptide Chains and Transition from Helix Structure to Random Coil Structure Using ATR-FTIR, Raman and ${ }^{1} \mathrm{HNMR}$ Spectroscopies. J Biomol Res Ther 5: e146. doi:10.4172/2167-7956.1000e146

Copyright: (c) 2016 Heidari A. This is an open-access article distributed under the terms of the Creative Commons Attribution License, which permits unrestricted use, distribution, and reproduction in any medium, provided the original author and source are credited. 
Citation: Heidari A (2016) Study of Irradiations to Enhance the Induces the Dissociation of Hydrogen Bonds between Peptide Chains and Transition from Helix Structure to Random Coil Structure Using ATR-FTIR, Raman and ${ }^{1}$ HNMR Spectroscopies. J Biomol Res Ther 5: e146. doi:10.4172/2167-7956.1000e146

Page 2 of 3

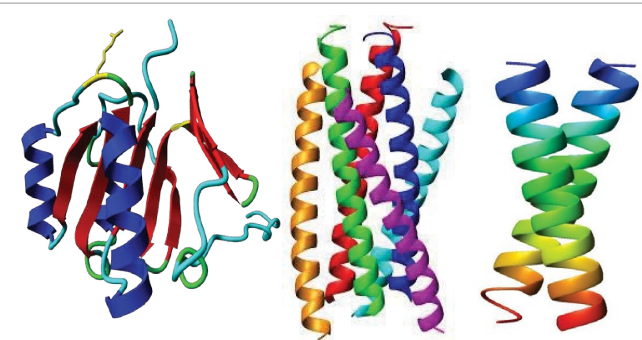

Figure 2: Different schematics of Collagen random coil structure [1].

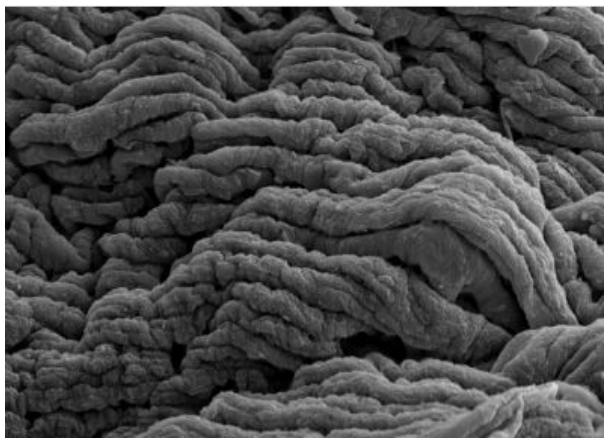

Figure 3: Scanning electron microscope (SEM) image of Collagen with triple helix structure with 200000x zoom.

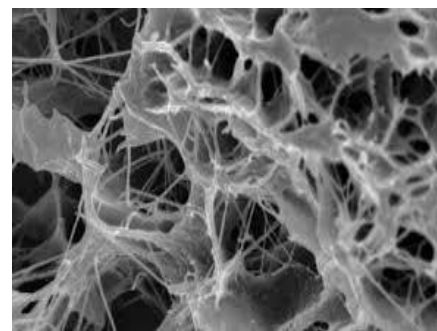

Figure 4: Transmission electron microscopy (TEM) image of Collagen with triple helix structure with $200000 x$ zoom.

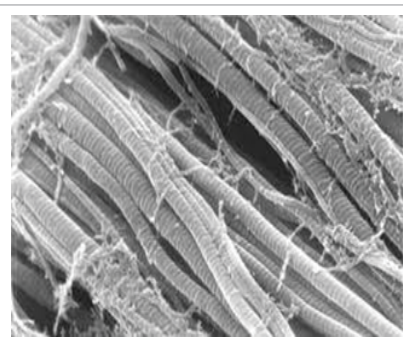

Figure 5: Scanning electron microscope (SEM) image of Collagen with random coil structure with $200000 \times$ zoom.

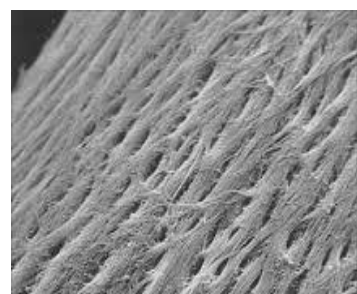

Figure 6: Transmission electron microscopy (TEM) image of Collagen with random coil structure with $200000 x$ zoom
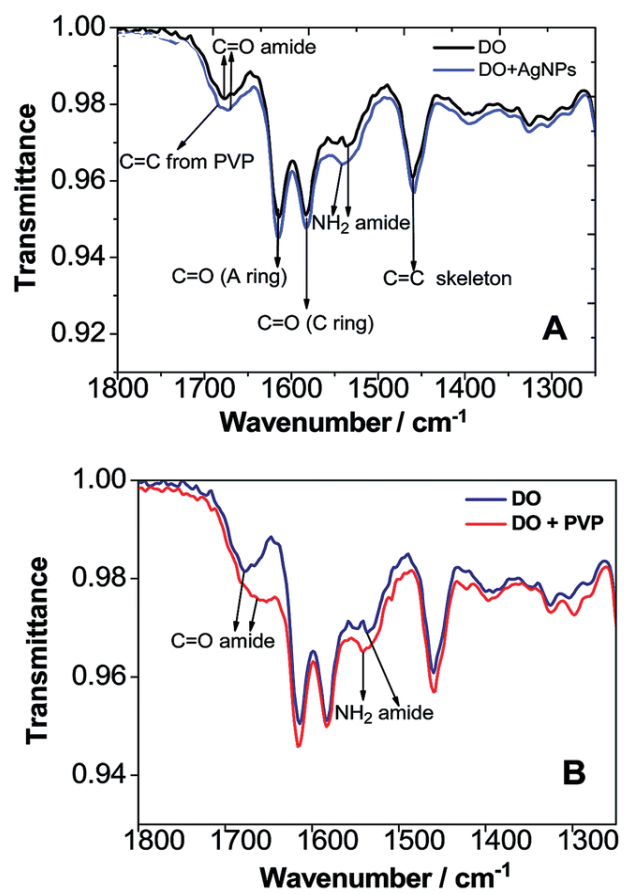

Figure 7: ATR-FTIR spectra of peptide chains transition from (a) helix structure to $(b)$ random coil structure.

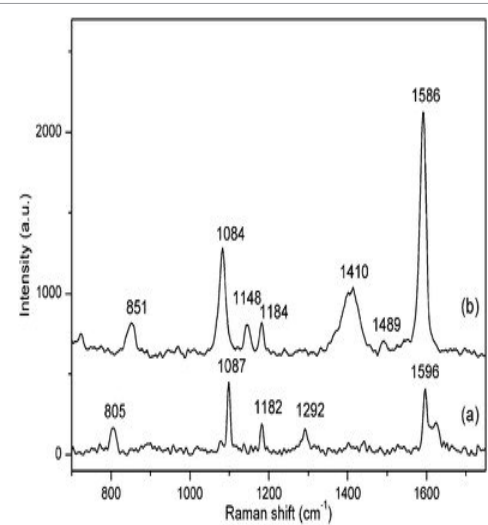

Figure 8: Raman spectra of peptide chains transition from (a) helix structure to (b) random coil structure.

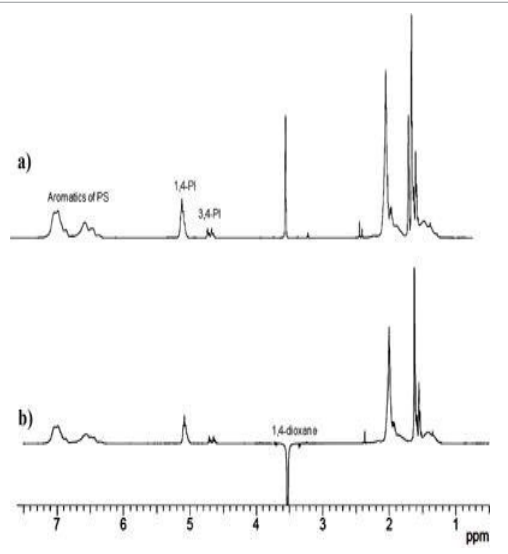

Figure 9: ${ }^{1} \mathrm{HNMR}$ spectra of peptide chains transition from (a) helix structure to (b) random coil structure. 
Citation: Heidari A (2016) Study of Irradiations to Enhance the Induces the Dissociation of Hydrogen Bonds between Peptide Chains and Transition from Helix Structure to Random Coil Structure Using ATR-FTIR, Raman and ${ }^{1} \mathrm{HNMR}$ Spectroscopies. J Biomol Res Ther 5: e146. doi:10.4172/2167-7956.1000e146

\section{References}

1. Heidari A (2012) Thesis submitted to the Faculty of the Chemistry, California South University (CSU), Irvine, California, The United States of America (USA) in Fulfillment of the Requirements for the Degree of Doctor of Philosophy (PhD) in Chemistry.

2. Guisasola EEB, Gutiérrez LJ, Salcedo RE, Garibotto FM, Andujar SA, et al (2016) Conformational transition of $A \beta_{42}$ inhibited by a mimetic peptide. A molecular modeling study using QM / MM calculations and QTAIM analysis. Computational and Theoretical Chemistry 1080: 56-65.

3. Minnen A, Bürmann F, Wilhelm L, Anchimiuk A, Diebold-Durand ML, et a I. (2016) Control of Smc Coiled Coil Architecture by the ATPase Heads Facilitates Targeting to Chromosomal ParB / parS and Release onto Flanking DNA. Cell Reports 14: 2003-2016.

4. Alves NS, Mendes YS, Souza TLF, Bianconi ML, Silva JL, et al. (2016) A biophysical characterization of the interaction of a hepatitis $C$ virus membranotropic peptide with micelles. Biochimica et Biophysica Acta (BBA) Proteins and Proteomics 1864: 359-371.

5. Zhang H, Sun J, Xin X, Xu W, Shen J, et al. (2016) Modulating self- assembly behavior of a salt-free peptide amphiphile (PA) and zwitterionic surfactant mixed system. Journal of Colloid and Interface Science 467: 43-50.

6. Barros SM, Whitaker SK, Sukthankar P, Avila LA, Gudlur S, et al. (2016) A review of solute encapsulating nanoparticles used as delivery systems with emphasis on branched amphipathic peptide capsules. Archives of Biochemistry and Biophysics 596: 22-42

7. Kumar ST, Leppert J, Bellstedt P, Wiedemann C, Fändrich M, et al. (2016) Solvent Removal Induces a Reversible $\beta$-to- $\alpha$ Switch in Oligomeric A $\beta$ Peptide. Journal of Molecular Biology 428: 268-273.

8. Chou S, Shao C, Wang J, Shan A, Xu L, et al. (2016) Short, multiple- stranded $\beta$-hairpin peptides have antimicrobial potency with high selectivity and salt resistance. Acta Biomaterialia 30: 78-93.

9. Ghosh A, Bera S, Shai Y, Mangoni ML, Bhunia A (2016) NMR structure and binding of esculentin-1a (1-21) $\mathrm{NH}_{2}$ and its diastereomer to lipopolysaccharide: Correlation with biological functions. Biochimica et Biophysica Acta (BBA) Biomembranes 1858: 800- 812
10. Patila M, Pavlidis IV, Kouloumpis A, Dimos K, Spyrou K, et al. (2016) Graphene oxide derivatives with variable alkyl chain length and terminal functional groups as supports for stabilization of cytochrome c. International Journal of Biological Macromolecules 84: 227-235.

11. Monteiro AA, Monteiro MR, Pereira RN, Diniz R, Costa AR, et al. (2016) Design of bio-based supramolecular structures through self-assembly of $\alpha$-lactalbumin and lysozyme. Food Hydrocolloids 58: 60-74.

12. Faccioli P, Beccara SA (2016) Computing Reaction Pathways of Rare Biomolecular Transitions using Atomistic Force-Fields. Biophysical Chemistry 208: 62-67.

13. Wang B, Yang W, McKittrick J, Meyers MA (2016) Keratin: Structure mechanical properties, occurrence in biological organisms, and efforts at bioinspiration. Progress in Materials Science 76: 229-318.

14. Cheng SY, Chou G, Buie C, Vaughn MW, Compton C, et al. (2016) Maximally asymmetric transbilayer distribution of anionic lipids alters the structure and interaction with lipids of an amyloidogenic protein dimer bound to the membrane surface. Chemistry and Physics of Lipids 196: 33-51.

15. Cheng TY, Chen MH, Chang WH, Huang MY, Wang TW (2013) Neural stem cells encapsulated in a functionalized self-assembling peptide hydrogel for brain tissue engineering. Biomaterials 34: 2005-2016.

16. Frazier SD, Srubar III WV (2016) Evaporation-based method for preparing gelatin foams with aligned tubular pore structures. Materials Science and Engineering: C 62: 467-473.

17. Roque A, Ponte I, Suau P (2016) Interplay between histone H1 structure and function. Biochimica et Biophysica Acta (BBA) - Gene Regulatory Mechanisms 1859: $444-454$

18. Croll TI, Smith BJ, Margetts MB, Whittaker J, Weiss MA, et al. (2016) HigherResolution Structure of the Human Insulin Receptor Ectodomain: Multi-Modal Inclusion of the Insert Domain. Structure 24: 469-476.

19. De Maria S, Ferrari G, Maresca P (2016) Effects of high hydrostatic pressure on the conformational structure and the functional properties of bovine serum albumin. Innovative Food Science and Emerging Technologies 33: 67-75. 